\title{
ALGEBRAIC CYCLES AND MOTIVIC GENERIC ITERATED INTEGRALS
}

\author{
Hidekazu Furusho AND Amir JAFARI
}

\begin{abstract}
Following [GGL1], we will give a combinatorial framework for motivic study of iterated integrals on the affine line. We will show that under a certain genericity condition these combinatorial objects yield to elements in the motivic Hopf algebra constructed in $[\mathrm{BK}]$. It will be shown that the Hodge realization of these elements coincides with the Hodge structure induced from the fundamental torsor of path of punctured affine line.
\end{abstract}

\section{Introduction}

Using cubical algebraic cycles, Bloch and Křiž [BK] constructed a category of mixed Tate motives over any field $F(\operatorname{MTM}(F)$ for short). They also constructed its Hodge realization and étale realization functor. Under the $\mathrm{K}(\pi, 1)$-conjecture (cf. §2), the desired formula

$$
\operatorname{Ext}_{M T M(F)}^{i}(\mathbf{Q}(0), \mathbf{Q}(n)) \cong g r_{i}^{\gamma} K_{2 n-i}(F)_{\mathbf{Q}}
$$

holds in this category. Here RHS is a graded quotient of the algebraic K-theory for $F$ with respect to $\gamma$-filtration. Our final aim is to construct a motivic fundamental group of the affine line minus finite set in $\operatorname{MTM}(F)$, which is equivalent to construct motivic iterated integrals. Our main results in this paper is a construction of motivic iterated integrals in generic positions.

In contrast there is another category of mixed Tate motives constructed for a field with characteristic 0 satisfying the Beilinson-Soulé vanishing conjecture, that is, $g r_{r}^{\gamma} K_{2 r-p}(F)_{\mathbf{Q}}=0$ for $p \leqslant 0$ with $(r, p) \neq(0,0)$. This category is a heart of $t$-structure given in [L1] of the sub-triangulated category generated by Tate motives inside the triangulated category of mixed motives over $F$ constructed by Voevodsky [V] and Levine [L3]. This category admits Hodge and étale realization [L3, H] and satisfies the desired formula above. Deligne and Goncharov [DG] constructed a motivic fundamental group of the affine line minus finite set in this category. This gives motivic iterated integrals in this category. As far as we know, the interrelationship between this category and that of Bloch-Křiž's mixed Tate motives are not known well although they are conjecturally equivalent. Deligne-Goncharov's construction of motivic fundamental group minus finite sets does not imply the existence of motivic iterated integrals in the category $\operatorname{MTM}(F)$ of Bloch-Křržs mixed Tate motives.

Received by the editors September 14, 2006. 
For $s_{0}, \ldots, s_{n+1} \in \mathbf{C}$ and a topological path $\gamma(t)(0 \leqslant t \leqslant 1)$ with $\gamma(0)=s_{0}$ and $\gamma(1)=s_{n+1}$. and $\gamma(t) \cap\left\{s_{i}\right\}=\emptyset$, the iterated integral is defined to be

$$
I t^{\gamma} \int_{s_{0}}^{s_{n+1}} \frac{d t}{t-s_{1}} \circ \cdots \circ \frac{d t}{t-s_{n}}:=\int_{0 \leqslant t_{1} \leqslant \cdots \leqslant t_{n} \leqslant 1} \frac{d \gamma\left(t_{1}\right)}{\gamma\left(t_{1}\right)-s_{1}} \wedge \cdots \wedge \frac{d \gamma\left(t_{n}\right)}{\gamma\left(t_{n}\right)-s_{n}} .
$$

For elements $s_{0}, \ldots, s_{n+1} \in \mathbb{A}_{F}^{1}$, we want to construct a motivic analogue of the above iterated integral which will be an element of the motivic Hopf algebra $\chi_{F}(n)$ (definition 2.1) and we denote it by $\mathbb{I}\left(s_{0} ; s_{1}, \ldots, s_{n} ; s_{n+1}\right)$. The special case with $s_{0}=0, s_{1}=1, s_{2}=\cdots=s_{n}=0, s_{n+1}=z$ which (up to a sign) represents $\mathrm{Li}_{n}(z):=\sum_{k=1}^{\infty} z^{k} / k^{n}$, was constructed in $[\mathrm{BK}]$. Unfortunately in order to have an admissible cycle we have to assume the following genericity condition;

Definition 1.1. The sequence $s_{0}, \ldots, s_{n+1}$ is generic if either $s_{0}=s_{n+1}$ holds or the non-zero terms do not repeat.

This generic condition is more enlarged than the condition given in [GGL2], where it is assumed that all $s_{i}$ 's are non-zero and distinct. With allowing zero to repeat we recover the expression given in $[\mathrm{BK}]$ for the polylogarithm, and moreover we get expressions for (several variable) multiple polylogarithm $L i_{n_{1}, \ldots, n_{m}}\left(z_{1}, \ldots, z_{m}\right)$ for the values $z_{i}$ such that $z_{i} \ldots z_{j} \neq 1$ for $1 \leqslant i \leqslant j \leqslant n$. In fact the method will work for certain sequences which are not generic, examples of such sequences are $\left(0 ; s_{1}, \ldots, s_{n} ; a\right)$ where at most two of $s_{i}$ 's are equal to 1 and the rest are zero. This will imply the existence of a motive giving double zeta value $\zeta(n, m)$ with $n+m$ : even for a number field $F(\S 8)$.

Our construction of motivic iterated integrals in generic position is based on the combinatorial framework of trees. We will consider a sum of trivalent trees with prescribed decorations and define motivic iterated integrals to be its corresponding cycle in Definition 4.4.

Theorem 1.2. If $F$ is a subfield of $\mathbf{C}$, the Hodge realization of the motivic iterated integral $(-1)^{n} \mathbb{I}\left(s_{0} ; s_{1}, \ldots, s_{n} ; s_{n+1}\right)$ agrees with the framed mixed Hodge Tate structure corresponding to the above iterated integral.

We will prove that motivic iterated integrals satisfy the usual properties of the iterated integrals, namely:

Theorem 1.3. Under the above genericity assumption for $a, b, s_{i}$ the elements $\mathbb{I}\left(a ; s_{1}, \ldots, s_{n} ; b\right)$ satisfy the following properties:

(1) Triviality: $\mathbb{I}(a ; b)=1$ and $\mathbb{I}\left(a ; s_{1}, \ldots, s_{n} ; a\right)=0$.

(2) Shuffle relation:

$$
\mathbb{I}\left(a ; s_{1}, \ldots, s_{n} ; b\right) \cdot \mathbb{I}\left(a ; s_{n+1}, \ldots, s_{n+m} ; b\right)=\sum_{\sigma \in S h(n, m)} \mathbb{I}\left(a ; s_{\sigma(1)}, \ldots, s_{\sigma(n+m)} ; b\right) .
$$

Here $\operatorname{Sh}(n, m)$ is the set of bijections $\tau:\{1, \ldots, n+m\} \rightarrow\{1, \ldots, n+m\}$ satisfying $\tau(1)<\cdots<\tau(n), \tau(n+1)<\cdots \tau(n+m)$.

(3)Path composition:

$$
\mathbb{I}\left(a ; s_{1}, \ldots, s_{n} ; b\right)=\sum_{k=0}^{n} \mathbb{I}\left(a ; s_{1}, \ldots, s_{k}, c\right) \cdot \mathbb{I}\left(c ; s_{k+1}, \ldots, s_{n} ; b\right) .
$$


(4) Antipode relation: $\mathbb{I}\left(a ; s_{1}, \ldots, s_{n} ; b\right)=(-1)^{n} \mathbb{I}\left(b ; s_{n}, \ldots, s_{1} ; a\right)$.

(5) Coproduct formula:

$$
\Delta \mathbb{I}\left(a ; s_{1}, \ldots, s_{n} ; b\right)=\sum \mathbb{I}\left(a ; s_{i_{1}}, s_{i_{2}}, \ldots, s_{i_{k}} ; b\right) \otimes \prod_{j=0}^{k} \mathbb{I}\left(s_{i_{j}} ; s_{i_{j}+1}, \ldots, s_{i_{j+1}-1} ; s_{i_{j+1}}\right)
$$

where the sum is over all indices $0=i_{0}<i_{1}<\cdots<i_{k}<i_{k+1}=n+1$ and $s_{0}:=a$ and $s_{n+1}:=b$ and $k=0,1, \ldots$

The content of the paper is as follows. $\S 2$ contains a review of definition of cubical cycle complex and the motivic Hopf algebra $\chi_{F}$ of $[\mathrm{BK}]$. The definition of bar complex is recalled where we use a different sign convention from [BK]. $\S 3$ gives a combinatorial differential graded algebra (DGA) built out of rooted decorated trees. The main idea is due to Goncharov [G1]. A morphism from the generic part of this DGA to the cubical cycle DGA is given. This extends the definition given in [GGL1]. In $\S 4$ for a given tuple $\left(a_{0} ; a_{1}, \ldots, a_{n} ; a_{n+1}\right)$ in a finite subset $S$ of $\mathbb{A}^{1}(F)$, we give our proposed definition for motivic iterated integral $\mathbb{I}(\mathbf{a})$. It is built out of a specific cycle $\rho(\mathbf{a})$ defined using 3 -valent trees. (The relation between 3 -valent trees and iterated integrals were first studied by Goncharov in [G1] and [G3]). The crucial property is a formula for the differential of this cycle. Theorem 1.3 is proven in $\S 5$, using the definitions of the previous section. $\S 6$ and 7 involve the important calculation of Hodge realization for the motivic iterated integrals when the ground field is embedded inside $\mathbb{C}$. This calculation justifies the name motivic iterated integral for the elements $\mathbb{I}(\mathbf{a})$ constructed in $\S 4$. In $\S 8$ we give some complementary remarks for the non-generic case.

This work is an extension of [GGL1]. We have been informed that Goncharov, Gangl and Levin completed its subsequent paper [GGL2], a part of which overlap with a part of ours.

\section{Bloch-Křiž' category of mixed Tate motives}

A differential graded algebra (DGA) with Adams grading is a bi-graded $\mathbb{Q}$-vector space, $\mathcal{A}=\oplus \mathcal{A}^{n}(r)$ where $n \in \mathbb{Z}$ and $r \geqslant 0$, such that $\mathcal{A}^{n}(r)=0$ for $n>2 r$ and $\mathcal{A}(0)=\mathbb{Q}$, together with a product $\mathcal{A}^{n}(r) \otimes \mathcal{A}^{m}(s) \longrightarrow \mathcal{A}^{n+m}(r+s)$ that makes $\mathcal{A}$ into a graded commutative algebra with identity (the signs are contributed from the differential grading and not the Adams grading), and a differential $d: \mathcal{A}^{n}(r) \longrightarrow$ $\mathcal{A}^{n+1}(r)$ that satisfies the Leibniz rule.

We now recall the bar construction. For our future need we develop the theory in a more general setting. Let $A$ be a DGA with Adams grading and let $M$ be a right DG module over $A$ with a compatible Adams grading. Let $A^{+}=\oplus_{r>0} A(r)$. Define $B(M, A)=M \otimes\left(\otimes^{\bullet} A^{+}[1]\right)$. The elements of $B(M, A)$ are denoted by a bar notation as $m\left[a_{1}|\ldots| a_{r}\right]$ which has a degree equal to $\operatorname{deg}(m)+\operatorname{deg}\left(a_{1}\right)+\ldots \operatorname{deg}\left(a_{r}\right)-r$. The graded vector space $B(M, A)$ is a differential graded with total Adams grading. The differential $d$ is given as the sum of two differentials, the external and internal differentials:

$$
d_{e x t}\left(m\left[a_{1}|\ldots| a_{r}\right]\right)=d m\left[a_{1}|\ldots| a_{r}\right]+\sum_{i=1}^{r} J m\left[J a_{1}|\ldots| J a_{i-1}\left|d a_{i}\right| a_{i+1}|\ldots| a_{r}\right],
$$


and

$$
d_{\text {int }}\left(m\left[a_{1}|\ldots| a_{r}\right]\right)=J m \cdot a_{1}\left[a_{2}|\ldots| a_{r}\right]+\sum_{i=1}^{r-1} J m \cdot\left[J a_{1}|\ldots| J a_{i-1}\left|J a_{i} \cdot a_{i+1}\right| \ldots \mid a_{r}\right],
$$

where the operation $J$ is given by $J(a)=(-1)^{\operatorname{deg}(a)-1} a$ on the homogeneous elements. Note that $\operatorname{deg}(a)-1$ is the degree if $a$ in the shifted complex $A[1]$. If $M$ is the trivial DGA, we have a coproduct (where the empty tensor is 1 by convention)

$$
\Delta\left(\left[a_{1}|\ldots| a_{r}\right]\right)=\sum_{s=0}^{r}\left[a_{1}|\ldots| a_{s}\right] \otimes\left[a_{s+1}|\ldots| a_{r}\right] .
$$

Up to this point all the constructions work for a DGA which is not necessarily commutative. The product is defined only when both $M$ and $A$ are commutative DGA's by the shuffle

$$
m\left[a_{1}|\ldots| a_{r}\right] \cdot m^{\prime}\left[a_{r+1}|\ldots| a_{r+s}\right]:=\sum \operatorname{sgn}_{\mathbf{a}}(\sigma) m \cdot m^{\prime}\left[a_{\sigma(1)}|\ldots| a_{\sigma(r+s)}\right]
$$

where $\sigma$ runs over the $(r, s)$-shuffles (an $(r, s)$-shuffle is a permutation $\sigma$ on $1, \ldots, r+s$ such that $\sigma^{-1}(1)<\cdots<\sigma^{-1}(r)$ and $\left.\sigma^{-1}(r+1)<\cdots<\sigma^{-1}(r+s)\right)$ and the sign is obtained by giving $a_{i}$ 's weights $\operatorname{deg}\left(a_{i}\right)-1$. These data make $B(A):=B(k, A)$ into a commutative differential Hopf algebra with an Adams grading. Taking $H^{0}$ with respect to the differential gives a commutative graded Hopf algebra $H^{0} B(A)$.

Following [BK], we now introduce the cubical cycle complex. Let $F$ be a field and denote $\square_{F}:=\mathbb{P}_{F}^{1} \backslash\{1\}$. Then the permutation group on $n$ letters the symmetric group $\mathfrak{S}_{n}$ acts on $\square_{F}^{n}$ and also we have an action of $(\mathbb{Z} / 2)^{n}$ given by $\epsilon \cdot\left(x_{1}, \ldots, x_{n}\right)=$ $\left(x_{1}^{\epsilon_{1}}, \ldots, x_{n}^{\epsilon_{n}}\right)$ where $\epsilon \in\{1,-1\}^{n}$. Therefor we have an action of $G_{n}:=(\mathbb{Z} / 2)^{n} \rtimes \mathfrak{S}_{n}$ on $\square_{F}^{n}$. Let $\operatorname{Alt}_{n} \in \mathbb{Q}\left[G_{n}\right]$ be the element $\left|G_{n}\right|^{-1} \sum_{g \in G_{n}} \operatorname{sgn}(g) g$ where for $g=$ $(\epsilon, \sigma) \in G_{n}$, sign is defined by $\left(\prod_{i} \epsilon_{i}\right) \operatorname{sgn}(\sigma)$. We also define a face of $\square_{F}^{n}$ as a subset defined by setting certain coordinates equal zero or infinity. We are now prepared to define the DGA with an Adams grading $\mathcal{N}=\mathcal{N}_{F}$ :

$$
\mathcal{N}^{n}(r):=\operatorname{Alt}_{2 r-n} Z\left(\square_{F}^{2 r-n}, r\right) .
$$

Notice that although $r \geqslant 0, n$ can be negative. Here $Z\left(\square_{F}^{n}, r\right)$ denotes the $\mathbb{Q}$-span of the admissible codimension $r$ subvarieties (i.e. closed and integral subschemes that intersect all the faces of codimension $\geqslant 1$ properly, i.e. in codimension $r$ ) of $\square_{F}^{n}$. The product structure is given by $Z_{1} \cdot Z_{2}:=\operatorname{Alt}\left(Z_{1} \times Z_{2}\right)$. The differential $d: \mathcal{N}^{n}(r) \longrightarrow \mathcal{N}^{n+1}(r)$ is given by $d=\sum_{i=1}^{2 r-n}(-1)^{i-1}\left(\partial_{0}^{i}-\partial_{\infty}^{i}\right)$ where for $c=0, \infty$, $\partial_{c}^{i}$ is obtained by the pull-back of the cycles under the inclusions $\square_{F}^{2 r-n-1} \hookrightarrow \square_{F}^{2 r-n}$ given by letting the $i^{\text {th }}$ coordinate equal to $c$. Using the results of [B1],[B2] and [L2] it is shown in $[\mathrm{BK}]$ that:

$$
\mathrm{H}^{n}(\mathcal{N}(r)) \cong \mathrm{CH}^{r}(F, 2 r-n) \otimes \mathbb{Q} \cong g r_{r}^{\gamma} K_{2 r-n}(F)_{\mathbb{Q}} .
$$

Here $\mathrm{CH}^{r}(F, n)$ denotes the Bloch's higher Chow group of $\operatorname{Spec}(F)$.

Definition 2.1. ([BK]) The category $\operatorname{MTM}(F)$ of mixed Tate motives over $F$ is the category of finite dimensional graded comodules over the motivic Hopf algebra, the graded commutative Hopf algebra $\chi_{F}:=\mathrm{H}^{0} B\left(\mathcal{N}_{F}\right)$. More explicitly a mixed Tate motive over $F$ is a graded finite dimensional $\mathbb{Q}$-vector space $M$ with a linear map 
$\nu: M \longrightarrow M \otimes \chi_{F}$ such that it respects the grading and $(i d \otimes \Delta)(\nu(a))=(\nu \otimes i d)(\nu(a))$ and $(i d \otimes \epsilon)(\nu(a))=a$ where $\Delta$ and $\epsilon$ are the coproduct and the counit of $\chi_{F}$.

The $\mathrm{K}(\pi, 1)$-conjecture says that the complex $\mathcal{N}_{F}$ should be quasi-isomorphic to the complex of its Sullivan 1-minimal model. This is stronger than the Beilinson-Soulé's vanishing conjecture saying that the complex should be cohomologically connected. Under the $\mathrm{K}(\pi, 1)$-conjecture, we have

$$
\operatorname{Ext}_{M T M(F)}^{i}(\mathbf{Q}(0), \mathbf{Q}(n)) \cong H^{i}\left(\mathcal{N}_{F}(n)\right)
$$

(see $[\mathrm{BK}]$ and also $[\mathrm{KM}]$ ). Here $\mathbf{Q}(n)$ is a copy of $\mathbf{Q}$ sitting in degree $-n$. The equalities of (2.1) and (2.2) give the desired formula (1.1). As far as we know the validity of Beilinson-Soulé's vanishing conjecture is known for number fields, function fields of the curve with genus 0 defined over number field and their inductive limits whereas the $\mathrm{K}(\pi, 1)$-conjecture is open for all fields.

\section{Trees and cycles}

The graph complex was introduced in the seminal work of Kontsevich in $[\mathrm{K}]$. The differential given here first appeared in [G1]. This section is a complement to [GGL1].

Let $S$ be a set. We define the DGA $\mathcal{T}_{S}$ (with an Adams grading) of rooted $S$ decorated planar trees. A rooted $S$-decorated planar tree is a connected finite graph with no loops, such that each edge has exactly two vertex and no vertex has degree 2 . Each vertex of degree one is decorated by an element of the given set $S$ and one such vertex is distinguished as a root. Decorated vertices which are not a root are called the ends. External edges which are not with a root are called leaves. Furthermore such a tree has a given embedding into the plane. The embedding into the plane defines a canonical ordering on the edges which is obtained by going counter clockwise starting with the root edge. The root defines a direction on each edge which is the direction away from the root.

Definition 3.1. (Tree algebra $\mathcal{T}_{S}$ ). The free graded commutative algebra generated by the rooted $S$-decorated planar trees, where each tree has weight equal to its number of edges, is denoted by $\mathcal{T}_{S}$. There is a bi-grading where a rooted tree in $\mathcal{T}_{S}^{n}(r)$ has $r$ ends and $2 r-n$ edges.

For a rooted $S$ decorated planar tree $T$, with a given edge $e$, the contracted tree $T / e \in \mathcal{T}_{S}$ is defined as follows: If $e$ is an internal edge then $T / e$ is obtained by contracting the edge $e$, keeping the root and decoration and embedding in the plane as before. If $e$ is an external edge (i.e. an edge with a decorated vertex) then $T / e$ is obtained in several stages. First remove $e$ and its two vertices. Denote the connected components by $T_{1}, \ldots, T_{k}$ (in the order dictated by the embedding), add a vertex to the open edges of these trees and decorate it by the decoration of the vertex of $e$. For the trees that do not have a root, make this newly added vertex their root. $T / e$ is the product of rooted $S$ decorated trees $\epsilon(e) T_{1} \cdots T_{k}$. Here $\epsilon(e)$ is \pm 1 according to the orientation as we now explain. Each planar tree has a canonical ordering on its edges given by going counter clockwise on the edges starting from the root. The sign $\epsilon(e)$ is defined from comparing the ordering defined by $T_{1} \cdots T_{k}$ and by $T$ with the edge $e$ removed. If these two ordering differ by an odd permutation the sign is minus and otherwise it is plus. Two examples are given in Figure 1. 


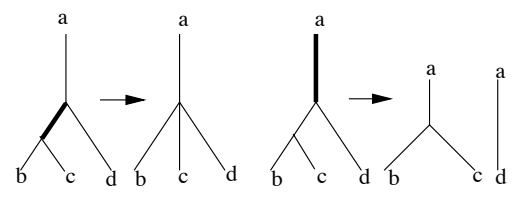

Figure 1. Two examples of contraction of edges

Definition 3.2. (Differential). For a rooted $S$ decorated planar tree $T$ in $\mathcal{T}_{S}$ the differential is given by $d T=\sum_{i=1}^{e(T)}(-1)^{i-1}\left(T / e_{i}\right)$. Here the ordering is given by the embedding by going counter clockwise starting from the root and $e(T)$ is the number of edges of $T$. This will be extended by Leibniz rule to all of $\mathcal{T}_{S}$.

The differential satisfies $d^{2}=0$, from which it follows that $\mathcal{T}_{S}$ becomes a commutative DGA.

Definition 3.3. Let $S$ and $S^{\prime}$ be two sets. The DGA of double decorated rooted trees $\mathcal{T}_{S, S^{\prime}}$ is the free graded algebra generated by rooted $S$-decorated planar trees together with a decoration of edges with values in $S^{\prime}$. We require three conditions:

(1) The two decoration of the leaves (one is of its edge and the other is of its external vertex) are distinct (we do not make this assumption for the root).

(2) For any edge decorated with $s^{\prime}$ and final vertex $v$, either $v$ is a decorated vertex or there is an edge starting from $v$ with the same edge decoration.

(3) Any path between two labeled vertex with the same label $s$, passes through the vertex of an edge decorated with $s$.

It is easy to check that the previous differential defines a differential structure on this algebra if we make the following modification: we do not collapse leaves whose vertex decoration is same as the edge decoration of their mother edge (its adjacent edge). Notice that the collapse of such an edge will produce a tree that does not satisfy the condition (1) above, nevertheless it does not produce any problem since this produces another tree with a root vertex decorated by same label as the root edge and the cycle associated to such a tree will be zero in the following definition. An example is given in Figure 2.

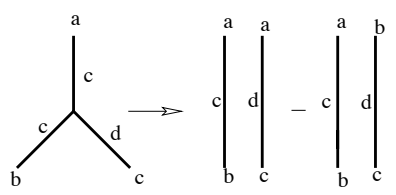

FiguRE 2. An example of differential of a double decorated tree

Bearing in mind the integral $\int_{a}^{c} \frac{d t}{t-b}$, for an oriented edge $e$ with origin vertex labeled by $a$ and the final vertex labeled by $b$ (they can be elements of $F$ or variables) and the edge labeled by $c$ define the function $f(e)=\frac{a-b}{c-b}$. We will define the following map which generalizes a construction of Gangl, Goncharov and Levin in [GGL1]: 
Definition 3.4. (Forest cycling map). Let $S$ be a non-empty subset of $\mathbb{A}^{1}(F)$. Decorate the internal vertices of elements of $\mathcal{T}_{S, S^{\prime}}$ by independent variables. Give each edge the direction that points away from the root. The forest cycling map associates an element $T$ of $\mathcal{T}_{S}^{n}(r)$ the following cycle of codimension $r$ inside $\square_{F}^{2 r-n}$ : $\rho(T)=\operatorname{Alt}_{2 r-n}\left(f\left(e_{1}\right), \ldots, f\left(e_{2 r-n}\right)\right)$. Here $e_{1}, \ldots, e_{2 r-n}$ are the edges of $T$ with the ordering induced from its embedding.

Let us explain this in more details. Let $I$ be the set of internal vertices. Then $\left(f\left(e_{1}\right), \ldots, f\left(e_{2 r-n}\right)\right)$ is a rational function from $\phi:\left(\mathbb{P}^{1}\right)^{I} \rightarrow\left(\mathbb{P}^{1}\right)^{2 r-n}$ and $\rho(T)$ is defined by the alternation of the following cycle: $\phi_{*}\left(\left(\mathbb{P}^{1}\right)^{I}\right) \cap \square_{F}^{2 r-n}$. This is a cycle of dimension equal to the number of internal vertices and hence the codimension equal to the number of end vertices.

Proposition 3.5. The map $\rho: \mathcal{T}_{S, S^{\prime}} \longrightarrow \mathcal{N}$ is a well-defined morphism of DGA's.

Proof. The compatibility with product: $\rho\left(T_{1} T_{2}\right)=\rho\left(T_{1}\right) \rho\left(T_{2}\right)$ is clear since $T_{1}$ and $T_{2}$ have different variables for their internal vertices. Therefore to show that $\rho(T)$ is admissible and $d \rho(T)=\rho(d T)$, we can assume that $T$ is connected. To show that $\rho(T)$ is admissible, first notice that this cycle does not intersect with the faces obtained by letting some coordinate take the value $\infty$. If the function associated to the edge $e$ from $a$ to $b$ with label $c$ is equal to $\infty$ then we are in one of the following two cases. Either $b$ is a variable and $b=c$, in which case the edge $e^{\prime}$ starting from $b$ with label $c$, gives the value 1 which is not in $\square_{F}$ (such an edge exists because of condition (2) above), or $a$ is a variable and $a=\infty$, in which case the edge $e^{\prime}$ ending at $a$ gives the value 1 . Now if we restrict the cycle $\rho(T)$ to the face with $i^{\text {th }}$-coordinate (corresponding to the edge $e_{i}$ from $a$ to $b$ ) equals zero, this means that we have to let $a=b$. Therefore we get $\rho\left(T / e_{i}\right)$. By induction on the number of edges we see that $\rho(T)$ is admissible and as a side we have proved that $d \rho(T)=\rho(d T)$. Notice that for the induction to work we need to assume validity of the condition (3) in Definition 3.3 , this is because a single edge with labels $a$ and $a$ gives the cycle $\{0\}-\{\infty\}$ which is not admissible.

Remark 3.6. The map $\rho$ factors through a quotient of $\mathcal{T}_{S, S^{\prime}}$ which we denote by $\tilde{\mathcal{T}}_{S, S^{\prime}}$. Two decorated trees $T_{1}$ and $T_{2}$ in $\mathcal{T}_{S, S^{\prime}}$ which can be transformed to each other by an automorphism of trees respecting the labels have the relation $T_{1}=\epsilon T_{2}$ where $\epsilon=1$ if the automorphism is an even permutation of the edges and $\epsilon=-1$ otherwise.

\section{Iterated Integrals and trees}

Let $S$ be a non-empty subset of $\mathbb{A}^{1}(F)$. For $a_{i} \in S$, for $i=0, \ldots, n+1$ define $\tilde{t}\left(a_{0} ; a_{1}, \ldots, a_{n}\right) \in \mathcal{T}_{S}^{1}(n)$ as the linear sum of all rooted planar 3 -valent trees with $n$ leaves decorated by $a_{1}, \ldots, a_{n}$ (in this order) and its root decorated by $a_{0}$. We define

$$
t\left(a_{0} ; a_{1}, \ldots, a_{n} ; a_{n+1}\right):=\tilde{t}\left(a_{0} ; a_{1}, \ldots, a_{n}\right)-\tilde{t}\left(a_{n+1} ; a_{1}, \ldots, a_{n}\right) .
$$

In this definition $t(a ; b)=0$ by our convention.

Proposition 4.1. The differential of $t\left(a_{0} ; a_{1}, \ldots, a_{n} ; a_{n+1}\right)$ is given by

$$
-\sum_{0 \leqslant i<j \leqslant n} t\left(a_{0} ; a_{1}, \ldots, a_{i}, a_{j+1}, \ldots ; a_{n+1}\right) t\left(a_{i} ; a_{i+1}, \ldots, a_{j} ; a_{j+1}\right)
$$

where the first term is obtained by removing $a_{i+1}$ up to $a_{j}$. 
Proof. The contraction of internal edges will cancel each other (cf. Figure 3).

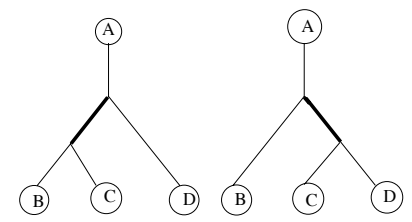

Figure 3. The contraction of the thick edge will give the same graph but with opposite signs

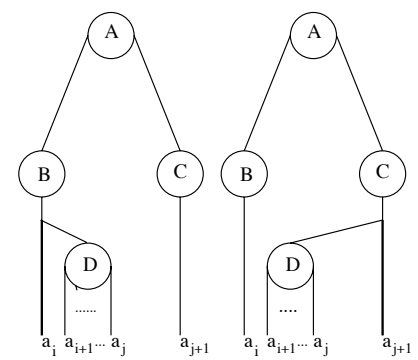

FiguRE 4. Grouping the trees

The term $-t\left(a_{0} ; \ldots, a_{i}, a_{j+1}, \ldots ; a_{n+1}\right) t\left(a_{i} ; a_{i+1}, \ldots, a_{j+1}\right)$ in the differential is obtained by grouping the trees in Figure 4 by contracting the edges corresponding to the labels $a_{i}$ and $a_{j+1}$.

Definition 4.2. (Admissible decomposition). An admissible decomposition of $\left(a_{0} ; a_{1}, \ldots, a_{n} ; a_{n+1}\right)$ is an ordered decomposition $D=P_{1} \cup \cdots \cup P_{k}$ of the regular polygon with vertices (with clockwise order) $a_{0}, \ldots, a_{n+1}$ into subpolygons $P_{i}$ by diagonals which do not intersect each other. The ordering should satisfy the following admissibility condition. If for $i<j, P_{i}$ and $P_{j}$ have a common edge and their union has vertices $a_{i_{1}}, \ldots, a_{i_{m}}$ in clockwise ordering starting with the vertex with the smallest index, then the edge $a_{i_{1}} a_{i_{m}}$ should belong to $P_{i}$. For a subpolygon $P$ with vertices $a_{i_{1}}, \ldots, a_{i_{m}}$ ordered as above we let $t(P):=t\left(a_{i_{1}} ; \ldots ; a_{i_{m}}\right)$, and for a decomposition $D=P_{1} \cup \cdots \cup P_{k}$ we let $t(D):=\left[t\left(P_{1}\right)|\ldots| t\left(P_{k}\right)\right] \in B(\mathcal{T})^{0}$, where $\mathcal{T}$ means $\mathcal{T}_{S}$.

Corollary 4.3. The element $T\left(a_{0} ; \ldots ; a_{n+1}\right):=\sum_{D} t(D) \in B(\mathcal{T})^{0}$ (where the sum is over all the admissible decomposition of a as above) has differential zero so defines an element in $H^{0} B(\mathcal{T})$ denoted again by $T(\mathbf{a})$.

Proof. The differential of $t(D)$ for an admissible decomposition $D=P_{1} \cup \cdots \cup P_{k}$ has two part, the external differential

$$
\sum_{i=1}^{k}\left[t\left(P_{1}\right)|\ldots| d t\left(P_{i}\right)\left|t\left(P_{i+1}\right)\right| \ldots \mid t\left(P_{k}\right)\right]
$$


and the internal differential

$$
\sum_{i=1}^{k-1}\left[t\left(P_{1}\right)|\ldots| t\left(P_{i}\right) t\left(P_{i+1}\right)|\ldots| t\left(P_{k}\right)\right]
$$

but according to proposition 4.1: $d t\left(P_{i}\right)=-\sum_{j} t\left(P_{i j}\right) t\left(P_{i j}^{\prime}\right)$ where the sum is taken over all possible division of the polygon $P_{i}$ into two sub-polygons $P_{i j}$ and $P_{i j}^{\prime}$ by a diagonal. This shows that the internal differentials for admissible decompositions of order $k$ will be canceled each other or by the external differentials of decompositions of order $k-1$. Therefore the differential of the sum over all possible decompositions vanishes.

Let $\mathcal{T}_{S}^{\prime}$ be the sub-DGA of $\mathcal{T}_{S}$ generated by admissible trees, i.e. those trees that the non-zero labels do not repeat. There is a map of DGA's dec : $\mathcal{T}_{S}^{\prime} \longrightarrow \mathcal{T}_{S,\{0,1\}}$ given by decorating the leaves with zero label by 1 and the rest of edges by 0 .

Definition 4.4. For generic sequence $\left(a_{0} ; a_{1}, \ldots, a_{n} ; a_{n+1}\right)$, i.e. a sequence with non repeating non-zero terms, define the motivic analogue of the iterated integral $(-1)^{n} \int_{a_{0}}^{a_{n+1}} \frac{d t}{t-a_{1}} \circ \cdots \circ \frac{d t}{t-a_{n}}$ by $\rho\left(\operatorname{dec}\left(T\left(a_{0} ; a_{1}, \ldots, a_{n} ; a_{n+1}\right)\right)\right)$. This is an element of $\chi_{F}(n)=\mathrm{H}^{0} B(\mathcal{N})(n)$ and will be denoted by $\mathbb{I}\left(a_{0} ; \ldots ; a_{n+1}\right)$. Here $\rho: \widetilde{\mathcal{T}}_{S,\{0,1\}} \longrightarrow$ $\mathcal{N}$ (for $\widetilde{\mathcal{T}}_{S,\{0,1\}}$ see Remark 3.6) and it induces a morphism $\rho: \mathrm{H}^{0} B\left(\widetilde{\mathcal{T}}_{S,\{0,1\}}\right) \longrightarrow$ $\mathrm{H}^{0} B(\mathcal{N})$.

In $\S 7$, it will be shown that the Hodge realization of this element agrees with the framed mixed Hodge Tate structure of the above iterated integral.

\section{Proof of the Theorem 1.3}

In this section we will prove theorem 1.3 from the introduction. It shows that $\mathbb{I}\left(a_{0} ; \ldots ; a_{n+1}\right)$ deserves to be called motivic iterated integral. In fact we have constructed $\mathbb{I}\left(a_{0} ; \ldots ; a_{n+1}\right)$ as the image (under the morphism $\rho \circ$ dec of an element $T\left(a_{0} ; \ldots ; a_{n+1}\right)$ in $B(\tilde{\mathcal{T}})^{0}$ with zero differential, where $\tilde{\mathcal{T}}$ stands for $\widetilde{\mathcal{T}}_{S,\{0,1\}}$. We will prove the identities of theorem 1.3 as identities in $B(\tilde{\mathcal{T}})^{0}$ with $\mathbb{I}$ replaced by $T$. This obviously implies the corresponding identities in $\mathrm{H}^{0} B(\mathcal{N})$.

We take $\mathbb{I}(a ; b)=1$ as definition. Since $t(a ; \ldots ; b)=0$ for $a=b$ it follows from the construction that $T(a ; \ldots ; b)=0$ for $a=b$. This proves the first part of the theorem.

We now prove the shuffle relation. Let $D=P_{1} \cup \cdots \cup P_{k}$ be an admissible decomposition of $\left(a ; s_{1}, \ldots, s_{n+m} ; b\right)$. For a permutation $\sigma$ of $s_{1}, \ldots, s_{n+m}$ we denote by $\sigma(D)$ the decomposition of $\left(a ; s_{\sigma(1)}, \ldots, s_{\sigma(n+m)} ; b\right)$ obtained by permuting the vertices of the polygons by $\sigma$, with the ordering remained as before. Suppose that one of the polygons $P_{i}$ of the decomposition $D$ has vertices (in order) $s_{i_{0}}, \ldots, s_{i_{l+1}}$ such that the sequence $i_{1}, \ldots, i_{l}$ is mixed, i.e. it has both numbers less than or equal to $n$ and numbers bigger than $n$. We show that

$$
\sum\left[t\left(P_{1}\right)|\cdots| t\left(\sigma\left(P_{i}\right)\right)|\cdots| t\left(P_{k}\right)\right]=0
$$

where the sum is taken over all shuffles $\sigma$ of $\left(i_{1}, \ldots, i_{l}\right)$ for the indices less than or equal to $n$ and those bigger than $n$. To prove this for any 3 -valent tree with decoration $s_{i_{1}}, \ldots, s_{i_{l}}$ (with this order or an order obtained by a shuffle of them) we define a dual tree that cancel it. The separating edge of a tree decorated by $s_{i_{1}}, \ldots, s_{i_{l}}$ means the 
first edge (using the ordering of the edges) that has the property that one of the sub-trees that grows out of this edge has decoration by indices less than or equal to $n$ and the other one has decoration by indices bigger than $n$. For a tree $T$ with the separating edge $e$ we define its dual the tree obtained from this tree by switching the two sub-trees of $e$, this is obtained by a shuffle on the decorating and clearly cancels $T$, in the modified algebra $\tilde{\mathcal{T}}$. Therefore we have to only consider the decompositions of $\left(a ; s_{\sigma(1)}, \ldots, s_{\sigma(n+m)} ; b\right)$ for $(n, m)$-shuffles $\sigma$, that are clean, i.e. each subdivision polygon with vertices $s_{i_{0}}, \ldots, s_{i_{l+1}}$ has the property that all the indices $i_{1}, \ldots, i_{l}$ are either bigger than $n$ or less than or equal to $n$. It is easy to see that the sum of $T(D)$ over this decompositions is equal to $T\left(a ; s_{1}, \ldots, s_{n} ; b\right) T\left(a ; s_{n+1}, \ldots, s_{n+m} ; b\right)$. This finishes the proof of the shuffle relation.

We now prove the path composition formula. For an admissible decomposition $D=P_{1} \cup \cdots \cup P_{m}$ of $\left(a ; s_{1}, \ldots, s_{n} ; b\right)$, let $P_{1}=P_{i_{0}}, P_{i_{1}}, \ldots, P_{i_{l}}$ be the sub-polygons with $b$ as a vertex. Using the trivial identity:

$$
t\left(s_{j_{1}} ; \ldots, s_{j_{p}} ; b\right)=t\left(s_{j_{1}} ; \ldots, s_{j_{p}} ; c\right)+t\left(c ; \ldots, s_{j_{p}} ; b\right)
$$

we can replace $t\left(P_{i_{p}}\right)$ as the sum of two terms $t\left(P_{i_{p}}^{\prime}\right)+t\left(P_{i_{p}}^{\prime \prime}\right)$ where $P_{i_{p}}^{\prime}$ is obtained by replacing the last vertex $b$ by $c$ in $P_{i_{p}}$ and similarly $P_{i_{p}}^{\prime \prime}$ is obtained by replacing the first vertex (the one with the smallest index) by $c$. Therefore we can write $t(D)$ as

$$
\sum_{k=0}^{l}\left[t\left(P_{1}^{\prime}\right)|\ldots| t\left(P_{i_{k-1}}^{\prime}\right)|\ldots| t\left(P_{i_{k}}^{\prime \prime}\right)\left|t\left(P_{i_{k}+1}\right)\right| \ldots \mid t\left(P_{m}\right)\right] .
$$

The $k^{t h}$ term of this sum when $P_{i_{k}}=\left(c ; s_{i}, \ldots ; b\right)$ belongs to the product

$$
\mathbb{I}\left(a ; s_{1}, \ldots, s_{i-1} ; c\right) \mathbb{I}\left(c ; s_{i}, \ldots ; b\right) .
$$

It is clear that this way we get an identification between the terms in $\mathbb{I}\left(a ; s_{1}, \ldots, s_{n} ; b\right)$ and $\sum_{i=1}^{n+1} \mathbb{I}\left(a ; s_{1}, \ldots, s_{i-1} ; c\right) \mathbb{I}\left(c ; s_{i}, \ldots, s_{n} ; b\right)$. This finishes the proof of path composition.

The proof of coproduct formula goes as follows. Recall the coproduct

$$
\Delta\left[a_{1}|\ldots| a_{n}\right]=\sum_{r=0}^{n}\left[a_{1}|\ldots| a_{r}\right] \otimes\left[a_{r+1}|\ldots| a_{n}\right] .
$$

Thus for a decomposition $D=P_{1} \cup \cdots \cup P_{m}$ we have

$$
\Delta t(D)=\sum_{r=0}^{m}\left[t\left(P_{1}\right)|\ldots| t\left(P_{r}\right)\right] \otimes\left[t\left(P_{r+1}\right)|\ldots| t\left(P_{m}\right)\right] .
$$

Taking the sum over all decompositions such that $P_{1} \cup \cdots \cup P_{r}$ is the polygon $\left(a ; s_{i_{1}}, \ldots, s_{i_{k}}, b\right)$ for a fixed sequence $0=i_{0}<i_{1}<\cdots<i_{k}<i_{k+1}=n+1$ and varying $r$ we get the term

$$
T\left(a ; s_{i_{1}}, s_{i_{2}}, \ldots, s_{i_{k}} ; b\right) \otimes \prod_{j=0}^{k} T\left(s_{i_{j}} ; s_{i_{j}+1}, \ldots, s_{i_{j+1}-1} ; s_{i_{j+1}}\right)
$$

in the coproduct. This finishes the proof of coproduct formula.

To prove the antipode relation note that the mirror dual of a tree with $n$ ends is equal to that tree time $(-1)^{n-1}$ in the modified algebra $\tilde{\mathcal{T}}$, so if we switch $a$ and $b$ as well a sign of $(-1)^{n}$ will appear. This finishes proof of the theorem 1.3. 


\section{Review of the Hodge realization}

In this section we review the construction of Bloch-Křiž for associating a framed MHTS to an element of $\mathrm{H}^{0} B(\mathcal{N})$. In fact for our application it is only enough to recall $\S 8$ of $[\mathrm{BK}]$ where they give the Hodge realization for $\mathrm{H}^{0} B\left(\mathcal{N}^{\prime}\right)$ for a particular sub-DGA $\mathcal{N}^{\prime}$ of $\mathcal{N}$.

Let $\omega(n, r)$ be a collection of real oriented subvarieties of $\left(\mathbb{P}^{1}\right)^{n}$ of codimension $2 r$. Assume that for $S_{1} \in \omega(n, r)$ and $S_{2} \in \omega(m, s), S_{1} \times S_{2} \in \omega(n+m, r+s)$, and for $\sigma \in G_{n}=(\mathbb{Z} / 2)^{n} \rtimes \mathfrak{S}_{n}, \sigma\left(S_{1}\right) \in \omega(n, r)$. Moreover assume that the intersection of an element of $\omega(n, r)$ with a hyperplane $t_{i}=0$ or $\infty$ belongs to $\omega(n-1, r)$. Define a DGA with an Adams grading by:

$$
\mathcal{D}^{n}(r):=\operatorname{Alt} \underline{\lim _{2 r-2 n}}\left(S \cup \mathcal{J}^{2 r-n}, \mathcal{J}^{2 r-n}\right)
$$

where $\mathcal{J}^{n}$ is the union of all the codimension 1 hyper planes of $\left(\mathbb{P}^{1}\right)^{n}$ obtained by letting one coordinate equal to 1 . The limit is taken over all $S \in \omega(2 r-n, r)$. This has a natural structure of DGA (cf.[BK] §8).

Definition 6.1. The pair $\left(\mathcal{N}^{\prime}, \mathcal{D}\right)$ as above, is called admissible if the following conditions hold:

(1) The Adams graded pieces $\mathrm{H}^{0} B\left(\mathcal{N}^{\prime}\right)(r)$ are finite dimensional $\mathbb{Q}$-vector spaces.

(2) All the support subvarieties of the elements of $\mathcal{N}^{\prime}$ belong to $\omega(*, *)$. Hence there is a natural morphism of DGA's: $\sigma: \mathcal{N}^{\prime} \longrightarrow \mathcal{D}$ given by the fundamental class.

(3) The map $\lambda_{0}: \mathcal{D} \longrightarrow \mathbb{C}[x]$ sending an element $c \in \mathcal{D}^{0}(n)$ to $(2 \pi i)^{n}\left(\int_{c} \omega_{2 n}\right) \cdot x^{n}$ and $\mathcal{D}^{i}$ for $i \neq 0$ to zero, is a well-defined morphism of DGA's. Here $\omega_{n}=(2 \pi i)^{-n} \frac{d z_{1}}{z_{1}} \wedge$ $\cdots \wedge \frac{d z_{n}}{z_{n}}$ and $\mathbb{C}[x]$ is a DGA with trivial differential and concentrated in degree zero with an Adams grading given by powers of $x$.

(4) If $\sum_{i}\left[a_{1}^{i}|\ldots| a_{r_{i}}^{i}\right] \in B\left(\mathcal{N}^{\prime}\right)^{0}$ has zero differential, then the element $\sum_{i} \sigma\left(a_{1}^{i}\right)\left[a_{2}^{i}|\ldots| a_{r_{i}}^{i}\right] \in B\left(\mathcal{D}, \mathcal{N}^{\prime}\right)^{1}$ is in $d\left(B\left(\mathcal{D}, \mathcal{N}^{\prime}\right)^{0}\right)$.

(5)The map $\alpha: \mathrm{H}^{0}\left(\mathcal{D} / \mathcal{N}^{\prime}(r)\right) \longrightarrow \mathrm{H}_{2 r}\left(\left(\mathbb{P}^{1} \backslash\{0, \infty\}\right)^{2 r} \cup N(r) \cup \mathcal{J}^{2 r}, \mathcal{J}^{2 r}\right) / \operatorname{Im} \gamma$ preserves integration of $\omega_{2 r}$. Here $N(r)$ is the union of codimension $r$ algebraic subvarieties of $\square_{\mathbb{C}}^{2 r}$ that intersect all the faces properly and $\gamma$ is the map:

$$
\gamma: \oplus_{H} \mathrm{H}_{2 r}\left(\mathcal{J}^{2 r}, H\right) \longrightarrow \mathrm{H}_{2 r}\left(\left(\mathbb{P}^{1} \backslash\{0, \infty\}\right)^{2 r} \cup N(r) \cup \mathcal{J}^{2 r}, \mathcal{J}^{2 r}\right)
$$

induced by inclusion, and $H$ runs over hyperplanes with one coordinate equal to one.

Let us quickly recall the notions from mixed Hodge-Tate structures (MHTS's). A MHTS $H$ is a finite dimensional $2 \mathbb{Z}$-graded $\mathbb{Q}$-vector space $H_{d R}=\oplus_{n} H_{2 n}$ together with a $\mathbb{Q}$-subspace $H_{B}$ of $H_{d R} \otimes \mathbb{C}$ such that for all $m$ :

$$
\operatorname{Im}\left(H_{B} \cap\left(\bigoplus_{n \leqslant m} H_{2 n} \otimes \mathbb{C}\right) \stackrel{\text { Proj }}{\longrightarrow} H_{2 m} \otimes \mathbb{C}\right)=(2 \pi i)^{m} H_{2 m} .
$$

A morphism $f$ is a graded morphism $f_{d R}$ from $H_{d R}$ to $H_{d R}^{\prime}$ such that $f_{d R} \otimes 1$ : $H_{d R} \otimes \mathbb{C} \longrightarrow H_{d R}^{\prime} \otimes \mathbb{C}$ sends $H_{B}$ to $H_{B}^{\prime}$, this induced map is denoted by $f_{B}$. The notion of framed MHTS was introduced by Beilinson, Goncharov, Schechtman and Varchenko in [BGSV]. This will give a concrete way of thinking about the Hopf algebra of the coordinate ring of the tannakian Galois group. A framing on a MHTS $H$ is the choice of a frame vector $v \in H_{2 r}$ and a coframe vector $\hat{v} \in \operatorname{Hom}\left(H_{2 s}, \mathbb{Q}\right)$. Two 
framed MHTS $(H, v, \hat{v})$ and $\left(H^{\prime}, v^{\prime}, \hat{v}^{\prime}\right)$ such that for an integer $n$ there is a morphism from $n$-Tate twisting $H(n) \longrightarrow H^{\prime}$ that respects the frames, are called equivalent. This relation generates an equivalence relation. The equivalence classes of framed MHTS is denoted by $\chi_{M H T S}$. This is graded where $[(H, v, \hat{v})]$ where $v \in H_{2 r}$ and $\hat{v} \in \operatorname{Hom}\left(H_{2 s}, \mathbb{Q}\right)$ has degree $r-s$. It is easily shown that the negative weights vanish. The product

$$
[(H, v, \hat{v})] \cdot\left[\left(H^{\prime}, v^{\prime}, \hat{v}^{\prime}\right)\right]=\left[\left(H \otimes H^{\prime}, v \otimes v^{\prime}, \hat{v} \otimes \hat{v}^{\prime}\right)\right]
$$

and the coproduct

$$
\Delta=\bigoplus_{m} \Delta_{m}: \quad \Delta_{m}[(H, v, \hat{v})]=\sum_{i}\left[\left(H, v, \hat{b}_{i}\right)\right] \otimes\left[\left(H, b_{i}, \hat{v}^{\prime}\right)\right]
$$

make $\chi_{M H T S}$ into a Hopf algebra. Here $b_{i}$ is a basis for $H_{2 m}$ and $\hat{b}_{i}$ is the dual basis for $\operatorname{Hom}\left(H_{2 m}, \mathbb{Q}\right)$. It can be shown that the category of finite dimensional graded comodules over $\chi_{M H T S}$ is equivalent to the category of MHTS's.

Under the conditions explained above on $\mathcal{D}$ Bloch and Křiž prove that:

Theorem $6.2([\mathrm{BK}])$. The graded $\mathbb{Q}$-vector space $H^{0} B\left(\mathcal{N}^{\prime}\right)=\oplus_{r} H^{0} B\left(\mathcal{N}^{\prime}\right)(r)$ where $H^{0} B\left(\mathcal{N}^{\prime}\right)(r)$ means its degree $2 r$-part, together with the subspace defined by the image of the map $\lambda$ defined as composition

$$
H^{0} B\left(\mathcal{D}, \mathcal{N}^{\prime}\right) \stackrel{B\left(\lambda_{0}, i d\right)}{\longrightarrow} H^{0} B\left(\mathbb{C}[x], \mathcal{N}^{\prime}\right) \stackrel{x=1}{\longrightarrow} H^{0} B\left(\mathcal{N}^{\prime}\right)_{\mathbb{C}}
$$

is a pro-MHTS. We denote its sub-MHTS on $\oplus_{0 \leqslant r \leqslant n} H^{0} B\left(\mathcal{N}^{\prime}\right)(r)$ by $H\left(\mathcal{N}^{\prime}, n\right)$.

The realization map $\operatorname{Real}_{M H T S}^{\prime}: \mathrm{H}^{0} B\left(\mathcal{N}^{\prime}\right)(n) \rightarrow \chi_{M H T S}(n)$ is given by sending $a \in \mathrm{H}^{0} B\left(\mathcal{N}^{\prime}\right)(n)$ to the framed MHTS $H:=H\left(\mathcal{N}^{\prime}, n\right)$ whose frame is given by $a \in \mathrm{H}^{0} B\left(\mathcal{N}^{\prime}\right)(n)=H_{2 n}$ and whose coframe is induced from the augmentation isomorphism $\mathrm{H}^{0} B\left(\mathcal{N}^{\prime}\right)(0) \rightarrow \mathbb{Q}$. It is proven in $[\mathrm{BK}]$ that:

Theorem 6.3. The realization map Real ${ }_{M H T S}$ is morphism of graded Hopf algebras. It is equal to the composition of $H^{0} B\left(\mathcal{N}^{\prime}\right) \longrightarrow H^{0} B(\mathcal{N})$ with the general Hodge realization Real RHTS $_{1}: H^{0} B(\mathcal{N}) \longrightarrow \chi_{M H T S}$ constructed in $\S \boldsymbol{7}$ of $[\mathrm{BK}]$.

\section{Proof of Theorem 1.2}

In this section we assume that $F$ is a subfield of $\mathbb{C}$ and $S$ is a finite subset of $F$. We will calculate the Hodge realization of $\mathbb{I}(\mathbf{a}) \in \mathrm{H}^{0} B\left(\mathcal{N}^{\prime}\right)$, where $\mathbf{a}=$ $\left(a_{0} ; a_{1}, \ldots, a_{n} ; a_{n+1}\right)$ is a generic sequence in $S$. We will do this parallel to the special case with $a_{0}=0, a_{1}=1, a_{2}=\cdots=a_{n}=0, a_{n+1}=a \in F$ which is done in [BK]. Here $\mathcal{N}^{\prime}=\mathcal{N}_{\mathbf{a}}$ is the sub-DGA of $\mathcal{N}$ generated by the cycles $\rho \circ \operatorname{dec} \circ t\left(\mathbf{a}^{\prime}\right) \in \mathcal{N}^{1}(n)$ for all sub-sequences $\mathbf{a}^{\prime}$ of $\mathbf{a}$. Notice that by definition $\mathcal{N}^{\prime}$ is a minimal DGA, i.e. it is connected and $d\left(\mathcal{N}^{\prime}\right) \subseteq \mathcal{N}^{\prime+} \cdot \mathcal{N}^{\prime+}$.

Lemma 7.1. The graded Hopf algebra $H^{0} B\left(\mathcal{N}^{\prime}\right)$ is generated as a DGA by the elements $\mathbb{I}\left(\mathbf{a}^{\prime}\right)$ for all sub-sequences of $\mathbf{a}$.

Proof. It follows from Theorem 6.3 in $[\mathrm{KM}]$ because $\mathcal{N}^{\prime}=\mathcal{N}^{\prime}\langle 1\rangle$ by our construction.

Once and for all choose a path $\gamma$ with interior in $\mathbb{A}^{1}(\mathbb{C})-S$ from the tangential base point $a_{0}$ to the tangential base point $a_{n+1}$. For $1 \leqslant i \leqslant n$ we define intermediate cycles $\eta_{i}(\mathbf{a})$ inside $\square_{\mathbb{C}}^{2 n-i}$ with (real) dimension $2 n-i$. It is defined as follows. Consider the 
sum of all rooted planar forests with $i$ connected components and $n$ leaves, decorated by $a_{1}, \ldots, a_{n}$. Its roots are decorated by variables $\gamma\left(s_{1}\right), \ldots, \gamma\left(s_{i}\right)$ for $0 \leqslant s_{1} \leqslant \cdots \leqslant$ $s_{i} \leqslant 1$. Apply the morphism $\rho \circ$ dec to this which will give an oriented (real) cycle (with boundary) of dimension $2 n-i$ inside $\square_{\mathbb{C}}^{2 n-i}$. Let $\Gamma$ be a small disk around zero in $\square_{\mathbb{C}}$ with its canonical orientation. We define

$$
\tau_{i}(\mathbf{a})=\left(\delta \eta_{i}(\mathbf{a})\right) \cdot \Gamma \cdot(\delta \Gamma)^{i-1}+(-1)^{i} \eta_{i}(\mathbf{a}) \cdot(\delta \Gamma)^{i}
$$

where denotes the usual alternating product and $\delta$ denotes the topological boundary defined for a cycle $f\left(s_{1}, \ldots, s_{n}\right)$ by $(\delta f)\left(s_{1}, \ldots s_{n-1}\right)=-f\left(0, s_{1}, s_{2}, \ldots, s_{n-1}\right)+$ $f\left(s_{1}, s_{1}, s_{2}, \ldots, s_{n-1}\right)-\cdots+(-1)^{n} f\left(s_{1}, \ldots, s_{n-1}, s_{n-1}\right)+(-1)^{n+1} f\left(s_{1}, \ldots, s_{n-1}, 1\right)$. Finally denote $\xi_{\gamma}(\mathbf{a})=\sum_{k=1}^{n} \tau_{k}(\mathbf{a})$. From now on we will use $\rho$ instead of $\rho \circ \operatorname{dec} \circ t$ for the ease in typing. Let $\omega(*, *)$ be the subvarieties which support the cycles $\xi\left(\mathbf{a}^{\prime}\right) \rho\left(\mathbf{a}^{\prime \prime}\right)$ and $\rho\left(\mathbf{a}^{\prime}\right)$ for all subsequences of $\mathbf{a}^{\prime}$ of $\mathbf{a}$, here $\mathbf{a}^{\prime \prime}$ is the complement of $\mathbf{a}^{\prime}$ inside $\mathbf{a}$. Denote by $\mathcal{D}=\mathcal{D}_{\mathbf{a}}$ the corresponding DGA obtained from $\omega(*, *)$. Hence $\xi_{\gamma}(\mathbf{a}) \in \mathcal{D}^{0}(n)$.

Proposition 7.2. The differential of $\xi_{\gamma}(\mathbf{a})$ is given by the formula

$$
\rho(\mathbf{a})-\sum_{0 \leqslant i<j \leqslant n} \xi_{\gamma}\left(a_{0} ; \ldots, a_{i}, a_{j+1}, \ldots ; a_{n+1}\right) \rho\left(a_{i} ; \ldots ; a_{j+1}\right) .
$$

As in corollary 4.3 for an admissible decomposition $D=P_{1} \cup \cdots \cup P_{k}$ let $\xi_{\gamma}(D):=$ $\xi_{\gamma}\left(P_{1}\right)\left[\rho\left(P_{2}\right)|\ldots| \rho\left(P_{k}\right)\right] \in B\left(\mathcal{D}, \mathcal{N}^{\prime}\right)$.

Corollary 7.3. Define $Z_{\gamma}(\mathbf{a}):=1 \cdot[\mathbb{I}(\mathbf{a})]+\sum_{D} \xi_{\gamma}(D)$ where the sum is over all admissible decompositions of $\mathbf{a}$. Then $d\left(Z_{\gamma}(\mathbf{a})\right)=0$. Therefore $Z_{\gamma}(\mathbf{a})$ defines an element of $H^{0} B\left(\mathcal{D}, \mathcal{N}^{\prime}\right)$.

Proof. Note that $d(1 \cdot[\mathbb{I}(\mathbf{a})])=-\sum_{D} \rho\left(P_{1}\right)\left[\rho\left(P_{2}\right)|\ldots| \rho\left(P_{k}\right)\right]$ where the sum is taken over all admissible decompositions $D=P_{1} \cup \cdots \cup P_{k}$ of $\mathbf{a}$. On the other hand for $D$ as above:

$$
\begin{aligned}
d_{e x t} \xi_{\gamma}(D)= & \left(d \xi_{\gamma}\left(P_{1}\right)\right)\left[\rho\left(P_{2}\right)|\ldots| \rho\left(P_{k}\right)\right]+\sum_{i=2}^{k} \xi_{\gamma}\left(P_{1}\right)\left[\rho\left(P_{2}\right)|\ldots| d \rho\left(P_{i}\right)|\ldots| \rho\left(P_{k}\right)\right] \\
= & \rho\left(P_{1}\right)\left[\rho\left(P_{2}\right)|\ldots| \rho\left(P_{k}\right)\right]-\sum_{j} \xi_{\gamma}\left(P_{1 j}\right) \rho\left(P_{1 j}^{\prime}\right)\left[\rho\left(P_{2}\right)|\ldots| \rho\left(P_{k}\right)\right] \\
& +\sum_{i=2}^{k} \xi_{\gamma}\left(P_{1}\right)\left[\rho\left(P_{2}\right)|\ldots| d \rho\left(P_{i}\right)|\ldots| \rho\left(P_{k}\right)\right] \\
d_{i n t} \xi_{\gamma}(D)= & \xi_{\gamma}\left(P_{1}\right) \rho\left(P_{2}\right)\left[\rho\left(P_{3}\right)|\ldots| \rho\left(P_{k}\right)\right] \\
& +\sum_{i=2}^{k-1} \xi_{\gamma}\left(P_{1}\right)\left[\rho\left(P_{2}\right)|\ldots| \rho\left(P_{i}\right) \rho\left(P_{i+1}\right)|\ldots| \rho\left(P_{k}\right)\right] .
\end{aligned}
$$

Since $d\left(\rho\left(P_{i}\right)\right)=-\sum_{j} \rho\left(P_{i j}\right) \rho\left(P_{i j}^{\prime}\right)$ for all divisions of $P_{i}$ into two admissible polygons $P_{i j}$ and $P_{i j}^{\prime}$. It will be seen that the third term in the external differential for $k$ will be canceled by the second term of the internal differential for $k+1$. The second term of the external differential for $k$ will be canceled by the first term of the internal differential for $k+1$. Finally the first term will be canceled by the differential of $1 \cdot[\mathbb{I}(\mathbf{a})]$. 
Proof of Proposition 7.2. We need the following two lemmas:

Lemma 7.4. The differential of $\eta_{k}(\mathbf{a})$ is given by:

$$
\delta \eta_{k+1}(\mathbf{a})-(-1)^{k} \sum_{0 \leqslant i<j \leqslant n} \eta_{k}\left(a_{0} ; a_{1}, \ldots, a_{i}, a_{j+1}, \ldots ; a_{n+1}\right) \rho\left(a_{i} ; \ldots ; a_{j+1}\right) .
$$

Lemma 7.5. The differential of $\delta \eta_{k}(\mathbf{a})$ is given by:

$$
(-1)^{k-1} \sum_{0 \leqslant i<j \leqslant n} \delta \eta_{k}\left(a_{0} ; \ldots, a_{i}, a_{j+1}, \ldots ; a_{n+1}\right) \rho\left(a_{i} ; \ldots ; a_{j+1}\right) .
$$

Assuming these lemmas, it follows:

$$
\begin{aligned}
d \tau_{k}(\mathbf{a})= & d \delta \eta_{k}(\mathbf{a}) \cdot \Gamma \cdot \delta \Gamma^{k-1}+(-1)^{k} \delta \eta_{k}(\mathbf{a}) \cdot \delta \Gamma^{k-1}+(-1)^{k} d \eta_{k}(\mathbf{a}) \cdot \delta \Gamma^{k} \\
= & -\sum_{0 \leqslant i<j \leqslant n}\left\{\delta \eta_{k}\left(a_{0} ; \ldots, a_{i}, a_{j+1}, \ldots ; a_{n+1}\right) \cdot \Gamma \cdot \delta \Gamma^{k-1}\right. \\
& \left.+(-1)^{k} \eta_{k}\left(a_{0} ; \ldots, a_{i}, a_{j+1}, \ldots ; a_{n+1}\right) \cdot \delta \Gamma^{k}\right\} \cdot \rho\left(a_{i} ; \ldots ; a_{j+1}\right) \\
& +(-1)^{k} \delta \eta_{k}(\mathbf{a}) \cdot \delta \Gamma^{k-1}+(-1)^{k} \delta \eta_{k+1}(\mathbf{a}) \cdot \delta \Gamma^{k} \\
= & -\sum_{0 \leqslant i<j \leqslant n} \tau_{k}\left(a_{0} ; \ldots, a_{i}, a_{j+1}, \ldots ; a_{n+1}\right) \cdot \rho\left(a_{i} ; \ldots ; a_{j+1}\right) \\
& +(-1)^{k} \delta \eta_{k}(\mathbf{a}) \cdot \delta \Gamma^{k-1}+(-1)^{k} \delta \eta_{k+1}(\mathbf{a}) \cdot \delta \Gamma^{k} .
\end{aligned}
$$

Adding for $k=1, \ldots, n$ we will get:

$$
d \xi_{\gamma}(\mathbf{a})=-\delta \eta_{1}(\mathbf{a})-\sum_{0 \leqslant i<j \leqslant n} \xi_{\gamma}\left(a_{0} ; \ldots, a_{i}, a_{j+1}, \ldots ; a_{n+1}\right) \cdot \rho\left(a_{i} ; \ldots ; a_{j+1}\right)
$$

but since $\delta \eta_{1}(\mathbf{a})=-\rho(\mathbf{a})$ this finishes the proof of proposition 7.2 modulo the two lemmas.

Proof of lemma 7.4. To calculate differential of $\eta_{k}(\mathbf{a})$ we have to calculate the differential of the sum over all 3 -valent forests with $k$ components decorated by $a_{1}, \ldots, a_{n}$ for the ends and $\gamma\left(s_{1}\right), \ldots, \gamma\left(s_{k}\right)$ for the roots, where $s_{i}$ 's are variables so that $0 \leqslant s_{1} \leqslant \cdots \leqslant s_{k} \leqslant 1$. To calculate this differential we have to consider the contraction of the edges. As in the proof of proposition 4.1 the only edges that contribute are external edges. The contraction of the roots after applying the morphism $\rho: \mathcal{T} \longrightarrow \mathcal{N}$ will give all the terms in $\delta \eta_{k+1}(\mathbf{a})$, except the terms corresponding to $s_{1}=0$, i.e. $\gamma\left(s_{1}\right)=a_{0}$ and $s_{k}=1$, i.e. $\gamma\left(s_{k}\right)=a_{n+1}$. The contraction of the leaves can be grouped like in the proof of proposition 4.1, and this together with two missing terms in $\delta \eta_{k}(\mathbf{a})$ will give

$$
(-1)^{k-1} \sum_{0 \leqslant i<j \leqslant n} \eta_{k}\left(a_{0} ; \ldots, a_{i}, a_{j+1}, \ldots ; a_{n+1}\right) \cdot \rho\left(a_{i} ; \ldots ; a_{j+1}\right) .
$$

This finishes the proof of lemma 7.4. 
Proof of lemma 7.5. From lemma 7.4 it follows that

$$
\begin{aligned}
d \delta \eta_{k+1}(\mathbf{a})= & (-1)^{k} d\left(\sum_{0 \leqslant i<j \leqslant n} \eta_{k}\left(a_{0} ; \ldots, a_{i}, a_{j+1}, \ldots ; a_{n+1}\right) \cdot \rho\left(a_{i} ; \ldots ; a_{j+1}\right)\right) \\
= & (-1)^{k} \sum_{0 \leqslant i<j \leqslant n}\left\{\delta \eta_{k+1}\left(a_{0} ; \ldots, a_{i}, a_{j+1}, \ldots ; a_{n+1}\right) \cdot \rho\left(a_{i} ; \ldots ; a_{j+1}\right)\right. \\
& \left.+(-1)^{k+1} d^{\prime}\left(\eta_{k}\left(a_{0} ; \ldots, a_{i}, a_{j+1}, \ldots ; a_{n+1}\right) \cdot \rho\left(a_{i} ; \ldots ; a_{j+1}\right)\right)\right\}
\end{aligned}
$$

Here $d^{\prime}$ is defined as the differential on $\rho$ but its defined by:

$$
d^{\prime} \eta_{k}\left(a_{0} ; \ldots ; a_{m+1}\right):=\sum_{0 \leqslant i<j \leqslant m} \eta_{k}\left(a_{0} ; \ldots, a_{i}, a_{j+1}, \ldots ; a_{m+1}\right) \cdot \rho\left(a_{i} ; \ldots ; a_{j+1}\right) .
$$

Now it follows from anti-commutativity that:

$$
\sum_{0 \leqslant i<j \leqslant n} d^{\prime}\left(\eta_{k}\left(a_{0} ; \ldots, a_{i}, a_{j+1}, \ldots ; a_{n+1}\right) \cdot \rho\left(a_{i} ; \ldots ; a_{j+1}\right)\right)=0,
$$

so lemma 7.5 is proved.

Lemma 7.6. Assume $a_{0} \neq a_{1}$ and $a_{n} \neq a_{n+1}$. The image of $\xi_{\gamma}(\mathbf{a}) \in \mathcal{D}^{0}(n)$ under the map $\lambda_{0}$ defined in definition 6.1 is given by the iterated integral:

$$
(-x)^{n} \int_{\gamma} \frac{d t}{t-a_{1}} \circ \cdots \circ \frac{d t}{t-a_{n}} .
$$

Furthermore $\lambda Z_{\gamma}(\mathbf{a}) \in H^{0} B\left(\mathcal{N}_{\mathbb{C}}^{\prime}\right)$ ( $\lambda$ was defined in theorem 6.2) is:

$$
\sum \int_{\gamma} \frac{d t}{t-a_{i_{1}}} \circ \ldots \frac{d t}{t-a_{i_{k}}} \prod_{j=0}^{k} \mathbb{I}\left(a_{i_{j}} ; \ldots ; a_{i_{j+1}}\right),
$$

where the sum is taken over all indices $0=i_{0}<i_{1}<\cdots<i_{k}<i_{k+1}=n+1$ with $k=0,1,2, \ldots$.

Proof. For reasons of type, the only term in $\sum_{k=1}^{n} \tau_{k}(\mathbf{a})$ which contributes in $\lambda_{0}\left(\xi_{\gamma}(\mathbf{a})\right)$ is $\tau_{n}(\mathbf{a})$ which is $\tau_{n}(\mathbf{a})=\left(\delta \eta_{n}(\mathbf{a})\right) \cdot \Gamma \cdot(\delta \Gamma)^{n-1}+(-1)^{n} \eta_{n}(\mathbf{a}) \cdot(\delta \Gamma)^{n}$. The first part of this sum gives zero integral for reasons of type. Note that $\eta_{n}(\mathbf{a})$ is $\operatorname{Alt}\left(f\left(s_{1}, a_{1}\right), \ldots, f\left(s_{n}, a_{n}\right)\right)$, where

$$
f\left(s_{i}, a_{i}\right)= \begin{cases}1-\frac{\gamma\left(s_{i}\right)}{a_{i}} & \text { if } a_{i} \neq 0 \\ \gamma\left(s_{i}\right) & \text { if } a_{i}=0 .\end{cases}
$$

Therefore

$$
\int_{\eta_{n}(\mathbf{a})} \frac{d z_{1}}{z_{1}} \wedge \cdots \wedge \frac{d z_{n}}{z_{n}}=\int_{\gamma} \frac{d t}{t-a_{1}} \circ \cdots \circ \frac{d t}{t-a_{n}} .
$$

Note that this integral is convergent due to the assumption of the lemma. This shows that

$$
x^{n}(2 \pi i)^{-n} \int_{\tau_{n}(\mathbf{a})} \frac{d z_{1}}{z_{1}} \wedge \cdots \wedge \frac{d z_{2 n}}{z_{2 n}}=(-x)^{n} \int_{\gamma} \frac{d t}{t-a_{1}} \circ \cdots \circ \frac{d t}{t-a_{n}} .
$$


This proves the first part of the lemma. To prove the second part, note that if in the set of all admissible decompositions one fixes $P_{1}=\left(a_{0}, a_{i_{1}}, \ldots, a_{i_{k}}, a_{n+1}\right)$ then

$$
\sum_{D}\left[\rho\left(P_{2}\right)|\ldots| \rho\left(P_{k}\right)\right]=\prod_{j=0}^{k} \mathbb{I}\left(a_{i_{j}} ; \ldots ; a_{i_{j+1}}\right) .
$$

This together with the first part of the lemma, letting $x=1$ finishes the proof.

Lemma 7.7. Let $\mathbf{a}=\left(a_{0} ; a_{1}, \ldots ; a_{n+1}\right)$ be a generic sequence in $S$ such that $a_{0} \neq a_{1}$ and $a_{n} \neq a_{n+1}$. The pair $\left(\mathcal{N}_{\mathbf{a}}, \mathcal{D}_{\mathbf{a}}\right)$ defined above is admissible in the sense of the definition 6.1 .

Proof. Since $\mathcal{N}^{\prime}$ is finitely generated, property (1) is clear. Property (2) follows from the definition of $\mathcal{D}$. To prove property (4) we can only consider elements $\mathbb{I}\left(\mathbf{a}^{\prime}\right)$ according to the lemma 7.1, and for these elements corollary 7.3 implies (4). Properties (3) and (5) follow from the same techniques as [BK], using a Stokes formula and the assumption on the convergence of the iterated integral, namely $a_{0} \neq a_{1}$ and $a_{n} \neq a_{n+1}$.

The MHTS on the pro-unipotent fundamental torsor $\Pi^{u n i}\left(\mathbb{A}^{1}-S ; a_{0}, a_{n+1}\right)$ is given by

$$
\begin{gathered}
\Pi_{d R}\left(\mathbb{A}^{1}-S ; a_{0}, a_{n+1}\right):=\mathbb{Q}\left\langle\left\langle X_{s}\right\rangle\right\rangle_{s \in S} \quad\left(\operatorname{weight}\left(X_{s}\right)=-2\right) \\
\Pi_{B}\left(\mathbb{A}-S ; a_{0} ; a_{n+1}\right):=\operatorname{Im}\left(\Pi^{\text {uni }}\left(\mathbb{A}^{1}-S ; a_{0}, a_{n+1}\right) \stackrel{\Phi}{\longrightarrow} \mathbb{C}\left\langle\left\langle X_{s}\right\rangle\right\rangle_{s \in S}\right) .
\end{gathered}
$$

Here the map $\Phi$ is defined by $\gamma \mapsto \sum\left(\int_{\gamma} \frac{d t}{t-s_{1}} \circ \cdots \circ \frac{d t}{t-s_{n}}\right) X_{s_{1}} \cdots X_{s_{n}}$ and the sum is taken over $n=0,1, \ldots$ and $s_{i} \in S$.

The Hodge analogue of the iterated integral $\int_{\gamma} \frac{d t}{t-a_{1}} \circ \cdots \circ \frac{d t}{t-a_{n}}$ is the framed MHTS given above for $\Pi:=\Pi^{u n i}\left(\mathbb{A}^{1}-S ; a_{0}, a_{n+1}\right)$, together with frames $1 \in \Pi_{0}$ and $\left(X_{a_{1}} \cdots X_{a_{n}}\right)^{\prime} \in \operatorname{Hom}\left(\Pi_{-2 n}, \mathbb{Q}\right)$. Here $\left\{\left(X_{s_{1}} \cdots X_{s_{m}}\right)^{\prime}\right\}_{s_{i} \in S}$ is the dual basis. We denote this by $\mathbb{I}^{\mathcal{H}}\left(a_{0} ; a_{1}, \ldots, a_{n} ; a_{n+1}\right)$.

Proof of Theorem1.2. First assume that $a_{0} \neq a_{1}$ and $a_{n} \neq a_{n+1}$. By lemma $7.7\left(\mathcal{N}^{\prime}, \mathcal{D}\right)$ is admissible, hence we can use the realization map $\operatorname{Real}_{M H T S}^{\prime}$. Let $\left(J_{\mathbf{a}}, \mathbb{I}(\mathbf{a}), \epsilon\right):=\operatorname{Real}_{M H T S}^{\prime}(\mathbb{I}(\mathbf{a}))$. We define a map $\Pi(n) \longrightarrow J_{\mathbf{a}}$ by

$$
X_{b_{1}} \cdots X_{b_{k}} \mapsto(-1)^{k} \sum \prod_{j=0}^{k} \mathbb{I}\left(a_{i_{j}} ; a_{i_{j}+1}, \ldots ; a_{i_{j+1}}\right)
$$

where the sum is taken over all indices $0=i_{0}<i_{1}<\cdots<i_{k}<i_{k+1}=n+1$ such that $a_{i_{j}}=b_{j}$. This obviously extends to a graded linear map from $\Pi(n) \longrightarrow J_{\mathbf{a}}$ which respects the corresponding frames. We need to show that for a path $\gamma$ from $a_{0}$ to $a_{n+1}$

$$
\sum \int_{\gamma} \frac{d t}{t-a_{i_{1}}} \circ \ldots \frac{d t}{t-a_{i_{k}}} \prod_{j=0}^{k} \mathbb{I}\left(a_{i_{j}} ; \ldots ; a_{i_{j+1}}\right),
$$

where the sum is taken over all indices $0=i_{0}<i_{1}<\cdots<i_{k}<i_{k+1}=n+1$ with $k=0,1,2, \ldots$, belongs to the Betti space of $J_{a}$. But this element is the image of $Z_{\gamma}(\mathbf{a})$ under the map $\lambda$ according to the lemma 7.6. This completes the proof of the equivalence of the two framed MHTS's. 
We now deal with the divergent iterated integrals. Note that by our construction $\mathbb{I}(0 ; 0 ; 1)=0$, therefore $\mathbb{I}\left(a_{0} ; a_{0} ; a_{1}\right)$ and $\mathbb{I}\left(a_{0} ; a_{1} ; a_{1}\right)$ have correct Hodge realization corresponding to the tangential base $\frac{\partial}{\partial t}$ for the canonical coordinate $t$ of $\mathbb{A}^{1}$. Therefore using the shuffle relation for

$$
\mathbb{I}(a_{0} ; \underbrace{a_{0}, \ldots, a_{0}}_{p} ; a_{n+1}) \cdot \mathbb{I}\left(a_{0} ; a_{1}, \ldots, a_{n} ; a_{n+1}\right) \cdot \mathbb{I}(a_{0} ; \underbrace{a_{n+1}, \ldots, a_{n+1}}_{q} ; a_{n+1})
$$

where $a_{0} \neq a_{1}$ and $a_{n} \neq a_{n+1}$, and induction on $p+q$ it follows that the divergent iterated integral

$$
\mathbb{I}(a_{0} ; \underbrace{a_{0}, \ldots, a_{0}}_{p}, a_{1}, \ldots, a_{n}, \underbrace{a_{n+1}, \ldots, a_{n+1}}_{q} ; a_{n+1})
$$

can be written as a linear combination of convergent iterated integrals. Since we have a same story in the Hodge side, with exact same formulas, it follows that the Hodge realization of $\mathbb{I}(\mathbf{a})$ is the framed MHTS associated to the iterated integral $(-1)^{n} \int_{a_{0}}^{a_{n+1}} \frac{d t}{t-a_{1}} \circ \cdots \circ \frac{d t}{t-a_{n}}$.

Our results gives the following examples.

Example 7.8. (1) For $a, b, c \in \mathbb{C}$ in generic position, the tree in the figure 5 gives a framed mixed Tate motive of the integral $\int_{a}^{b} \frac{d t}{t-c}$.

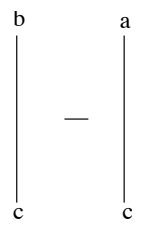

Figure 5. The element of $H^{0}\left(B\left(\mathcal{T}_{S}\right)\right)$ corresponding to $\int_{a}^{b} \frac{d t}{t-c}$.

(2) For $a, b, c, d \in \mathbb{C}$ in generic position, the tree in the figure 6 gives a framed mixed Tate motive of the integral It $\int_{a}^{b} \frac{d t}{t-c} \circ \frac{d t}{t-d}$.

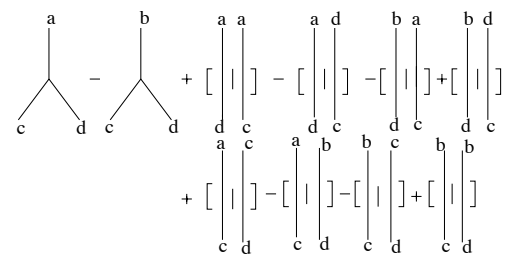

Figure 6. The tree corresponding to $\int_{a}^{b} \frac{d t}{t-c} \circ \frac{d t}{t-d}$.

Example 7.9. (1) For $a \in \mathbb{C}$ and $k \in \mathbb{N}$ the framed mixed Tate motive associated to the polylogarithm $L i_{k}(a)=\sum_{n} \frac{a^{n}}{n^{k}}$ is given by the following cycles [BK]: $\rho_{k}(a)+$ $\left[\rho_{k-1}(a) \mid \rho_{1}(1-a)\right]+\left[\rho_{k-2}(a)\left|\rho_{1}(1-a)\right| \rho_{1}(1-a)\right]+\cdots+\left[\rho_{1}(a)\left|\rho_{1}(1-a)\right| \cdots \mid \rho_{1}(1-a)\right]$ where $\rho_{k}(a)$ is $(-1)^{\frac{k(k-1)}{2}}$ Alt $\left(x_{1}, \cdots, x_{k-1}, 1-x_{1}, 1-\frac{x_{2}}{x_{1}}, \cdots, 1-\frac{x_{k-1}}{x_{k-2}}, 1-\frac{a}{x_{k-1}}\right)$. 
(2) For $n, m \in \mathbb{N}$ and $a, b \in \mathbb{C}$ with $b \neq 1$ and $a b \neq 1$ the framed mixed Tate motive associated to the double polylogarithm $L i_{n, m}(a, b)=\sum_{0<k<l} \frac{a^{k} b^{l}}{k^{n} l^{m}}$ is given by the following cycles:

$$
\begin{aligned}
\sum_{k \geqslant 0, l \geqslant 0} & (-1)^{n+k}\left(\begin{array}{c}
n+m-k-l-2 \\
n-l-1
\end{array}\right) \rho_{a, b}(l, n+m-l-k-2, k)+\left[\rho_{b}(n) \mid \rho_{a}(m)\right] \\
& +(-1)^{n}\left(\begin{array}{c}
n+m-2 \\
n-1
\end{array}\right)\left[\operatorname{Alt}\{1-a b\} \mid \rho_{\frac{1}{a}}(n+m-1)-\rho_{b}(n+m-1)\right] \\
& +\sum_{i=1}^{m-1}(-1)^{n}\left(\begin{array}{c}
m+n-i-2 \\
m-i-1
\end{array}\right)\left[\rho_{a b}(i+1) \mid \rho_{\frac{1}{a}}(m+n-i-1)\right] \\
& +\sum_{j=1}^{n-1}(-1)^{n+j+1}\left(\begin{array}{c}
m+n-j-2 \\
n-j-1
\end{array}\right)\left[\rho_{a b}(j+1) \mid \rho_{b}(m+n-j-1)\right] .
\end{aligned}
$$

where $\rho_{a, b}(n, m, k)=$

$$
\begin{aligned}
& (-1)^{m+n} \operatorname{Alt}\left(1-\frac{1}{x_{1}}, 1-\frac{x_{1}}{x_{2}}, \ldots, 1-\frac{x_{k+n}}{x_{k+n+1}}, 1-a b x_{k+n+1}, 1-\frac{x_{k+1}}{x_{k+n+2}},\right. \\
& \left.\ldots, 1-\frac{x_{k+n+m}}{x_{k+n+m+1}}, 1-b x_{k+n+m+1}, x_{k+n+m+1}, x_{k+n+m}, \ldots, \widehat{x_{k+1}}, \ldots, x_{1}\right), \\
& \rho_{a}(n)=(-1)^{n-1} \operatorname{Alt}\left(1-\frac{1}{x_{1}}, 1-\frac{x_{1}}{x_{2}}, \ldots, 1-\frac{x_{n-2}}{x_{n-1}}, 1-a x_{n-1}, x_{n-1}, \ldots, x_{1}\right) .
\end{aligned}
$$

Because of the condition $b \neq 1$ and $a b \neq 1$, it does not give a cycle representing the double zeta value $\zeta(n, m)=\sum_{0<k<l} \frac{1}{k^{n} l^{m}}$. However in the following section we will construct it for $n+m$ : even when $F$ is a number field.

\section{Miscellaneous remarks}

In the previous sections for any sequence $a_{0}, a_{1}, \ldots, a_{n}, a_{n+1}$ of elements of $F$, an element $t\left(a_{0} ; a_{1}, \ldots, a_{n} ; a_{n+1}\right) \in \mathcal{T}_{F}^{1}(n)$ was constructed. The crucial property was its differential formula given in Proposition 4.1. From this one can construct an element $T\left(a_{0} ; a_{1}, \ldots, a_{n} ; a_{n+1}\right) \in \mathrm{H}^{0} B\left(\mathcal{T}_{F}\right)$. The problem of constructing motivic iterated integrals is that there is no natural morphism from $\mathcal{T}_{F} \longrightarrow \mathcal{N}$, whereas we have a morphism: $\mathcal{T}_{F, F} \longrightarrow \mathcal{N}$. Therefore one needs to have a lifting of $t\left(a_{0} ; \ldots ; a_{n+1}\right)$ to $\mathcal{T}_{F, F}$ that has the same differential formula. When no non-zero term repeats, such a lifting exists. Namely by given zero labels to all edges except the leaves with zero vertex label which get 1 as their edge label. We were unable to construct a canonical lifting for the general case. In this section we want to give some partial results that we obtained in this direction.

Lemma 8.1. There is a natural lifting of $t\left(a_{0} ; a_{1}, a_{2} ; a_{3}\right)$ in $\mathcal{T}_{F, F}$.

Proof. A 3-edge trivalent tree with root decorated by $a$ and the leaves decorated by $b$ and $c$, has the following lifting to $\mathcal{T}_{F, F}$ : if $(a, b, c)$ is generic then we label the edges as before. If $b=c$ then by symmetry it is zero. If $a=b$ and $b \neq c$ then we label the edge with vertex label $c$ by $b$ and the other two edges as usual. Similarly if $a=c$ and $b \neq c$, we label the edge with vertex label $b$ by $c$ and the other two edges 
as usual. It is easy to see that it defines an element of $\mathcal{T}_{F, F}$ and is compatible with the differential. Using this we can define the desired lifting of $t\left(a_{0} ; a_{1}, a_{2} ; a_{3}\right)$.

Lemma 8.2. Let $\mathbf{a}=\left(0 ; a_{1}, \ldots, a_{n} ; 1\right)$ be a sequence with at most two $a_{i}$ equal to 1 and the rest equal to zero. There is a lifting of $t(\mathbf{a})$ to $\mathcal{T}_{F,\{0,1\}}$ such that it satisfies the same differential equation as in proposition 4.1 .

Proof. Notice that the terms in the differential are admissible by the assumption on a. The only type of trees that are not admissible in $t(\mathbf{a})$ are those with root labeled by 1 and the two edges coming off the root edge, one is a leaf with vertex 1 and the other is an internal edge. To make this admissible we label this internal edge by 1 . This way the differential of the tree would not be changed and therefore the same differential equation holds for this lifting.

Example 8.3. With this admissible lifting we can apply the method of corollary 4.3. We therefore have an element of $\mathrm{H}^{0} B(\mathcal{N})$. It satisfies the same coproduct formula as for the double zeta framed motive constructed in [G3], therefore its difference with the true double zeta motive is in $\operatorname{Ext}^{1}(\mathbb{Q}(0), \mathbb{Q}(n))$, where $n$ is the weight of the framed object. In case $n$ is even and we are over a number field $F$ this extension is zero and we therefore get the true double zeta motive. To do this concisely we define the cycles $\rho(n, m, k)$. Here $n, m, k$ are non-negative integers. If $n+k>0$, they are given by:

$$
\begin{aligned}
& \rho(n, m, k)=(-1)^{m+n} \operatorname{Alt}\left(1-\frac{1}{x_{1}}, 1-\frac{x_{1}}{x_{2}}, \ldots, 1-\frac{x_{k+n}}{x_{k+n+1}}, 1-x_{k+n+1}, 1-\frac{x_{k+1}}{x_{k+n+2}},\right. \\
& \left.1-\frac{x_{k+n+2}}{x_{k+n+3}}, \ldots, 1-\frac{x_{k+n+m}}{x_{k+n+m+1}}, 1-x_{k+n+m+1}, x_{k+n+m+1}, \ldots, \widehat{x_{k+1}}, \ldots, x_{2}, x_{1}\right)
\end{aligned}
$$

and if $n=k=0$ :

$$
\begin{gathered}
\rho(0, m, 0)=(-1)^{m} \operatorname{Alt}\left(1-\frac{1}{x_{0}}, 1-x_{0}, \frac{x_{0}-x_{1}}{1-x_{1}}, 1-\frac{x_{1}}{x_{2}}, \ldots, 1-\frac{x_{m-1}}{x_{m}},\right. \\
\left.1-x_{m}, x_{m}, \ldots, x_{1}\right) .
\end{gathered}
$$

Using this notation the cycle associated to $\zeta(n, m)$ for $n+m$ an even integer is:

$$
\sum_{k \geqslant 0, l \geqslant 0}(-1)^{m-k-1}\left(\begin{array}{c}
n+m-k-l-2 \\
n-l-1
\end{array}\right) \rho(l, n+m-l-k-2, k) .
$$

To give an element of $\mathrm{H}^{0} B(\mathcal{N})$ representing $\zeta(n, m)$ for $n+m$ even we have:

$$
\begin{aligned}
\zeta(n, m)= & \sum_{k \geqslant 0, l \geqslant 0}(-1)^{m-k}\left(\begin{array}{c}
n+m-k-l-2 \\
n-l-1
\end{array}\right) \rho(l, n+m-l-k-2, k) \\
& +[\zeta(n) \mid \zeta(m)]+\sum_{i=1}^{m-1}(-1)^{m}\left(\begin{array}{c}
n+m-i-2 \\
m-i-1
\end{array}\right)[\zeta(i+1) \mid \zeta(m+n-i-1)] \\
& -\sum_{j=1}^{n-1}(-1)^{m+j}\left(\begin{array}{c}
m+n-j-2 \\
n-j-1
\end{array}\right)[\zeta(j+1) \mid \zeta(m+n-j-1)] .
\end{aligned}
$$

where $\zeta(n)$ is the following cycle:

$$
(-1)^{n-1} \operatorname{Alt}\left(1-\frac{1}{x_{1}}, 1-\frac{x_{1}}{x_{2}}, \ldots, 1-\frac{x_{n-2}}{x_{n-1}}, 1-x_{n-1}, x_{n-1}, \ldots, x_{1}\right) .
$$




\section{Acknowledgements}

The authors want to thank IAS for providing an ideal environment for research. We specially want to thank Herbert Gangl for answering our questions. We also thank Kasra Rafi for lots of advice and comments.

\section{References}

[BGSV] A. A. Beilinson, A. B. Goncharov, V. V. Schechtman, and A. N. Varchenko, Aomoto dilogarithms, mixed Hodge structures and motivic cohomology of pairs of triangles on the plane, The Grothendieck Festschrift, Vol. I, 135-172, Progr. Math., 86, Birkhaüser Boston, Boston, MA, 1990. [B1] S. Bloch, Algebraic cycles and higher K-theory, Adv. in Math. 61 (1986), no. 3, 267-304.

[B2] _ , The moving lemma for higher Chow groups, J. Algebraic Geom. 3 (1994), no. 3, 537568

[BK] S. Bloch and I. Křiž, Mixed Tate motives, Ann. of Math. (2) 140 (1994), no. 3, 557-605.

[DG] P. Deligne and A. B. Goncharov, Groupes fondamentaux motivique de Tate mixte, Ann. Sci. Éc. Norm. Sup, (4) 38 (2005), no. 1, 1-56.

[GGL1] H. Gangl, A. B. Goncharov, and A. Levini Multiple logarithms, algebraic cycles and trees, Frontiers in number theory, physics, and geometry. II, 759-774, Springer, Berlin, 2007.

[GGL2] Multiple polylogarithms, polygons, trees and algebraic cycles, Preprint. arXive:math.NT/0508066.

[G1] A. B. Goncharov. Multiple $\zeta$-values, Galois groups, and geometry of modular varieties, European Congress of Mathematics, Vol. I (Barcelona, 2000), 361-392, Progr. Math., 201, Birkhaüser, Basel, 2001.

[G2] _ Multiple polylogarithms and mixed Tate motives, Preprint in arXive:math. AG/0103059.

[G3] _ Galois symmetries of the fundamental groupoid and Non-commutative Geometry, Duke Math. J. 128 (2005), no. 2, 209-284.

[H] A. Huber, Mixed Motives and their Realizations in Derived Categories, LNM 1604, SpringerVerlag, 1995.

[K] M. Kontsevich, Formal (non) commutative symplectic geometry, The Gelfand Mathematical Seminars, 1990-1992, 173-187, Birkhäuser Boston, Boston, MA, 1993

[KM] I. Kriz, Igor and J. P. May, Operads, algebras, modules and motives, Asterisque No. 233 (1995), iv+145pp.

[L1] M. Levine, Tate motives and the vanishing conjectures for algebraic $K$-theory, Algebraic $K$ theory and algebraic topology (Lake Louise, AB, 1991), 167-188, NATO Adv. Sci. Inst. Ser. C Math. Phys. Sci., 407, Kluwer Acad. Publ., Dordrecht, 1993.

[L2] B Bloch's higher Chow groups revisited, K-theory (Strasbourg, 1992). Asterisque No. 226 (1994), 10, 235-320.

[L3] _ _ Mixed Motives, Math Surveys and Monographs 57, AMS (1998).

[V] V. Voevodsky, Triangulated categories of motives over a field. Cycles, transfers, and motivic homology theories, 188-238, Ann. of Math. Stud., 143, Princeton Univ. Press, Princeton, NJ, 2000.

Graduate School of Mathematics, Nagoya University, Nagoya, 464-8602, Japan

E-mail address: furusho@math.nagoya-u.ac.jp

Math Departments, Duke University, Durham, NC, 27708, USA

E-mail address: amir@math.duke.edu 